Gazi University
Journal of Science
$\mathrm{http} / /$ dergipark.gov.tr/gujs

\title{
Estimation of Mean of a Sensitive Quantitative Variable in Complex Survey: Improved Estimator and Scrambled Randomized Response Model
}

\author{
Iram SALEEM ${ }^{1}{ }^{(0)}$, Aamir SANAULLAH ${ }^{2}$, Nursel KOYUNCU ${ }^{3, *}$ (i) Muhammad HANIF $^{1}$ \\ ${ }^{1}$ National College of Business Administration and Economics Lahore, Pakistan \\ ${ }^{2}$ COMSATS Institute Of Information Technology, Lahore, Pakistan \\ ${ }^{3}$ Department of Statistics, Hacettepe University, Beytepe, Ankara, Turkey
}

\section{Highlights}

- This paper introduces a new Scrambled Randomized Response model.

- A simulation study is done to compare the efficiency of the proposed model.

- The results showed that the proposed model performs better than existing model.

Article Info

Received: $26 / 04 / 2018$

Accepted: 17/12/2019

\section{Keywords}

Scrambled Randomized Response

Sensitive Variable

Exponential Estimator

Simulation

\begin{abstract}
With the intention to control a true swapping between the efficiency and the privacy protection this paper introduces a scrambled randomized response (SRR) model to be alternative of Saha's scrambling mechanism. The basic initiative is to provide an assortment of the additive, the subtractive and the multiplicative models. The simulation and the empirical studies are provided for various sample sizes to compare the efficiency of the proposed model. The results obtained from simulation showed that the proposed model performs better than Pollock and Bek's additive model. Also, the proposed generalized estimator of mean has been studied using a new SRR model presented in this article and shown that the proposed estimator and its class of estimators are more efficient than existing estimators. It is also shown that gain in efficiency is more when the proposed SRR model is used. The efficiency of the proposed class of estimators over existing estimators using both models is also provided using real data and with a simulation study.
\end{abstract}

\section{INTRODUCTION}

In surveys having sensitive issues such as tax evasion, gambling, child abuse and drug misuse, we do not obtain trustworthy information easily from the respondents. To get reliable and valid information in such surveys, Warner [1] introduced a randomized response technique (RRT) to protect the privacy of respondents. This method is easy to use for gathering information about sensitive qualitative response variable, for example, to estimate the proportion of women in a community enduring induced abortions. Unfortunately, this proposed method is not applicable for sensitive quantitative response variable, for instance, to find out the average abortion number in a community. Greenberg et al. [2] and Pollock and Bek [3] extended the work of Warner [1] to obtain a reliable response for quantitative variables. Himmelfarb and Edgell [4] revisited the Pollock and Bek's [3] additive model and studied it in more detail for estimating the mean of a quantitative sensitive variable.

Further, Eichhorn and Hayre [5] introduced a multiplicative model for quantitative surveys. Eichhorn and Hayre [5] presented the concept of scrambled randomized response technique (SRR) in which the respondent provides a quantitative reply to the sensitive question asked and then response is multiplied to 
the random number generated by the respondents themselves from some randomized device. Further contribution and modifications in SRR technique by developing some new models, have been made by several authors such as, Saha [6], Diana and Perri [7,8]. Hussain [9] considered a subtractive SRR model which involves the respondent subtracting the scrambling variable from the answer to sensitive questions. Many different modifications in SRR technique to develop some new models have been made by several authors such as, Chen and Singh [10], Singh and Tarray [11] and Hussain and Al-Zahrani [12]. We can list recent important studies about scrambled response models under ranked set sampling (RSS) as follows: Bouza [13] considered some important scrambled response models under the use of simple random sampling, RSS and Rao-Hartley-Cochran designs. Bouza [14] examined scrambled responses in RSS. Bouza et al. [15] studied the an Optional Scrambling Randomized Response Modeling procedure under RSS design.

Several authors such as Sousa et al. [16], Gupta et al. [17] , Tarray and Singh [18] and Koyuncu et al. [19] presented different estimators to estimate the population mean of a sensitive study variable using a nonsensitive auxiliary variable.

Saha $[6,20]$ 's model can be considered when survey questions are extremely sensitive. In such a situation scrambling device provides a greater protection to the respondent but on the other hand efficiency of the estimators cannot be expected to be good. Therefore, this study aims to look into a possible alternative to Saha's approach that is more flexible in terms of correctness and secrecy protection. With intention to hold a right trade-off between the efficiency and secrecy protection, an attempt is made successfully to provide a blend of the three models, Pollock and Bek's [3] additive, Hussain's [9] subtractive and Eichhron and Hayre's [5] multiplicative models are provided and show that proposed model performs better. Further aim of the study is extended to show that the proposed estimator performs better than some existing estimators when either our proposed SRR model or the Pollock and Bek's model is used. The main objective is to provide the evidence that under the proposed SRR model the proposed estimators as well as the existing estimators, perform more efficiently. In Section2, a general SRR model is proposed with its properties. In Section3, an improved class of generalized estimators is discussed. Further, the proposed estimator is also shown to be an almost unbiased and the most efficient estimator. Also the optimal conditions in order to attain the minimum mean square errors are discussed. In Section4, a simulation study is used to show the dominance of the proposed procedure over existing Pollock and Bek's additive model and the efficiency of the proposed generalized estimator. The concluding remarks are given in Section5.

Pollock and Bek [3] introduced an additive model to obtain reliable answers for a quantitative sensitive variable under study. In this approach, the respondent is asked to sum of the sensitive value say $Y$ and the random scrambling value say $S$ from the known distribution, independent to $Y$. Suppose a random sample of size $n$ is drawn without replacement from a finite population $U=\left(U_{1}, U_{2}, \ldots, U_{N}\right)$. For the $i$ th unit $(\mathrm{i}=1,2, \ldots, \mathrm{N})$ let $Y_{i}$ and $S_{i}$ be the values of study and scrambling variable. Let $\bar{y}=\sum_{i=1}^{n} Y_{i} / n$ and $\bar{Y}=\sum_{i=1}^{N} Y_{i} / N$ be the sample mean and population mean of $Y$ respectively and $\bar{S}=\sum_{i=1}^{N} S_{i} / N$ denotes the population mean of $S$. We can define following expectations $E(S)=\bar{S}=0$ and $E(Y)=\bar{Y}$ The reported response following Pollock and Bek's [3] additive model is given by,

$$
Z_{P B}=Y+S
$$

for the $i$ th unit $(\mathrm{i}=1,2, \ldots, \mathrm{N}), Z_{i}$ denotes the sum of the sensitive value and the random scrambling value the estimated mean for $Z_{P B}$ can be estimated by 
$\hat{\mu}_{Z_{P B}}=\bar{z}=\frac{\sum_{i=1}^{n} Z_{i}}{n}$,

with variance

$\operatorname{Var}\left(\hat{\mu}_{Z_{P B}}\right)=\theta\left(S_{Y}^{2}+S_{S}^{2}\right)$

where $\theta=\frac{1}{n}-\frac{1}{N}, S_{y}^{2}=\frac{1}{N-1} \sum_{i=1}^{N}\left(Y_{i}-\bar{Y}^{2}\right)$ and $S_{s}^{2}=\frac{1}{N-1} \sum_{i=1}^{N}\left(S_{i}-\bar{S}^{2}\right)$ are the variances of $Y$ and $S$, respectively.

Eichhorn and Hayre [5] presented a multiplicative approach adapting the initial idea of Pollock and Bek [3] in depth and assuming another scrambling random variable $R$ with the mean and variance respectively as $E(R)=\bar{R}$ and $\operatorname{Var}(R)=S_{R}^{2}$. Then the observed scrambled response is

$Z_{E H}=Y R$

and the estimated mean of $\bar{Y}$ is

$\hat{\mu}_{Z_{E H}}=\frac{\bar{z}}{\bar{R}}$

where $\bar{z}_{E H}=\frac{\sum_{i=1}^{n} z_{E H(i)}}{n}$ is the sample mean of $n$ coded responses. The variance of $\hat{\mu}_{E H}$ is given by,

$\operatorname{Var}\left(\hat{\mu}_{Z_{E H}}\right)=\frac{1}{n}\left[S_{Y}^{2}+\bar{Y}^{2}\left(1+C_{Y}^{2}\right) C_{R}^{2}\right]$

where $C_{Y}^{2}=\frac{S_{y}^{2}}{\bar{Y}^{2}}$ and $C_{R}^{2}=\frac{S_{R}^{2}}{\bar{R}^{2}}$.

The auxiliary information can be used at both design and estimation stages to gain precision of the estimators of population parameters. Diana and Perri [7, 8] proposed some classes of estimators using a sensitive auxiliary variable. Likewise, different estimators to estimate the population mean of a sensitive study variable using a non-sensitive auxiliary variable were presented by several authors. Sousa et al. [16] presented a ratio estimator for sensitive study variable $(Y)$ using a non-sensitive auxiliary variable $\left(X_{1}\right)$. The estimator $t_{S}$ presented by Sousa et al. [16] is given by,

$t_{S}=\bar{z} \frac{\bar{X}_{1}}{\bar{x}_{1}}$

where $\bar{X}_{1}$ and $\bar{X}_{1}$ are the sample mean and population mean of non-sensitive auxiliary variable respectively. The MSE of $t_{S}$ is given by, 


$$
\operatorname{MSE}\left(t_{s}\right)=\theta \bar{Y}^{2}\left(C_{z}^{2}+C_{x_{1}}^{2}-2 C_{z} C_{x_{1}} \rho_{z x_{1}}\right)
$$

where $C_{z}$ and $C_{x_{1}}$ are the coefficient of variation of $Z$ and $X_{1}$ respectively. The ratio estimators are widely used when the correlation coefficient between the randomized response variable and auxiliary variable is strong and positive. The estimator $t_{S}$ provides better performance than usual unbiased mean estimator $\bar{z}$ when $\rho_{Z X_{1}}>\frac{C_{X_{1}}}{2 C_{Z}}$ and usual unbiased estimator is preferred over $t_{S}$ if $-\frac{C_{X_{1}}}{2 C_{Z}}<\rho_{z x}<\frac{C_{X_{1}}}{2 C_{Z}}$. Gupta et al. [17] proposed a regression-cum-ratio estimator is written as,

$t_{G}=\left[k_{1} \bar{z}+k_{2}\left(\bar{X}_{1}-\bar{x}_{1}\right)\right]\left(\frac{\bar{X}_{1}}{\bar{x}_{1}}\right)$

where $k_{1}$ and $k_{2}$ are constants.

$$
\begin{aligned}
& \operatorname{MSE}\left(t_{s}\right)=\left(k_{1}-1\right) \bar{Y}^{2}+k_{1}^{2} \bar{Y}^{2} \theta\left(C_{z}^{2}+3 C_{x_{1}}^{2}-4 C_{z} C_{x_{1}} \rho_{z x_{1}}\right)+k_{2}^{2} \bar{X}^{2} \theta C_{x_{1}}^{2} \\
& -2 k_{1} \bar{Y}^{2} \theta\left(C_{x_{1}}^{2}-C_{z} C_{x_{1}} \rho_{z x_{1}}\right)-2 k_{2} \bar{Y} \bar{X} \theta C_{x_{1}}^{2}-2 k_{1} k_{2} \overline{Y X} \theta\left(C_{z} C_{x_{1}} \rho_{z x_{1}}-2 C_{x_{1}}^{2}\right) .
\end{aligned}
$$

Koyuncu et al. [19] suggested two generalized regression-cum-exponential estimator using non-sensitive auxiliary variables. The first proposed estimator and its MSE are given by,

$$
\begin{aligned}
t_{K_{1}}=\left[w_{1} \bar{z}+\right. & \left.w_{2}\left(\bar{X}_{1}-\bar{x}_{1}\right)\right] \exp \left(\frac{\bar{X}_{1}-\bar{x}_{1}}{\bar{X}_{1}+\bar{x}_{1}}\right), \\
\operatorname{MSE}\left(\mathrm{t}_{K_{1}}\right)= & \left\{\overline{\mathrm{Z}}^{2}+\mathrm{w}_{1}^{2} \overline{\mathrm{Z}}^{2}\left(1-1\left(\mathrm{C}_{\mathrm{z}}^{2}+\mathrm{C}_{\mathrm{x}_{1}}^{2}-2 \mathrm{C}_{\mathrm{zx}_{1}}\right)\right)+\mathrm{w}_{2}^{2} \overline{\mathrm{X}}_{1}^{2} \mathrm{C}_{\mathrm{x} 1}^{2}\right. \\
& \left.+\mathrm{w}_{1} \overline{\mathrm{Z}}^{2}\left(1\left(\mathrm{C}_{\mathrm{zx}_{1}}+\frac{3}{4} \mathrm{C}_{\mathrm{x}_{1}}^{2}\right)-2\right)-\mathrm{w}_{2} \overline{\mathrm{Z}} \overline{\mathrm{X}}_{1} \mathrm{C}_{\mathrm{x} 1}^{2}+2 \mathrm{w}_{1} \mathrm{w}_{2} \overline{\mathrm{Z}} \overline{\mathrm{X}}_{1}\left(\mathrm{C}_{\mathrm{x}_{1}}^{2}-\mathrm{C}_{\mathrm{zx}_{1}}\right)\right\}
\end{aligned}
$$

Their second estimator and its MSE are given by,

$$
\begin{aligned}
t_{K_{2}}=\left[d_{1} \bar{z}+\right. & \left.d_{2}\left(\bar{X}_{1}-\bar{x}_{1}\right)+d_{3}\left(\bar{X}_{2}-\bar{x}_{2}\right)\right] \exp \left(\frac{\left(\bar{X}_{1}-\bar{x}_{1}\right)+\left(\bar{X}_{2}-\bar{x}_{2}\right)}{\left(\bar{X}_{1}+\bar{x}_{1}\right)+\left(\bar{X}_{2}+\bar{x}_{2}\right)}\right), \\
\operatorname{MSE}\left(t_{K_{2}}\right)= & \left\{\bar{Z}^{2}+d_{1} A-d_{2} B-d_{3} C+d_{1}^{2} D+d_{2}^{2} \bar{X}_{1}^{2} \theta C_{x_{1}}^{2}+d_{3}^{2} \bar{X}_{2}^{2} \theta C_{x 2}^{2}\right. \\
& \left.+2 d_{1} d_{2} F+2 d_{1} d_{3} G+2 d_{1} d_{3} \bar{X}_{1} \bar{X}_{2} \theta C_{x_{1} x_{2}}\right\}
\end{aligned}
$$

Where

$$
A=\bar{Z}^{2}\left(-2+\theta\left\{\frac{\bar{X}_{1} C_{z x_{1}}}{\left(\bar{X}_{1}+\bar{X}_{2}\right)}+\frac{\bar{X}_{2} C_{z x_{2}}}{\left(\bar{X}_{1}+\bar{X}_{2}\right)}-\frac{3 \bar{X}_{1}^{2} C_{x_{1}}^{2}}{4\left(\bar{X}_{1}+\bar{X}_{2}\right)^{2}}-\frac{6 \bar{X}_{1} \bar{X}_{2} C_{x_{1} x_{2}}}{4\left(\bar{X}_{1}+\bar{X}_{2}\right)^{4}}-\frac{3 \bar{X}_{2}^{2} C_{x_{2}}^{2}}{4\left(\bar{X}_{1}+\bar{X}_{2}\right)^{2}}\right\}\right)
$$




$$
\begin{aligned}
& B=\theta \frac{\bar{Z}}{\left(\bar{X}_{1}+\bar{X}_{2}\right)}\left(\bar{X}_{1}^{2} C_{x_{1}}^{2}+\bar{X}_{1} \bar{X}_{2} C_{x_{1} x_{2}}\right), C=\theta \frac{\bar{Z}}{\left(\bar{X}_{1}+\bar{X}_{2}\right)}\left(\bar{X}_{2}^{2} C_{x_{2}}^{2}+\bar{X}_{1} \bar{X}_{2} C_{x_{1} x_{2}}\right), \\
& D=\bar{Z}^{2}\left(1+\theta\left\{C_{z}^{2}+\frac{\bar{X}_{1}^{2} C_{x_{1}}^{2}}{\left(\bar{X}_{1}+\bar{X}_{2}\right)^{2}}+\frac{\bar{X}_{2}^{2} C_{x_{2}}^{2}}{\left(\bar{X}_{1}+\bar{X}_{2}\right)^{2}}-2 \frac{\bar{X}_{1} C_{z x_{1}}}{\left(\bar{X}_{1}+\bar{X}_{2}\right)}-2 \frac{\bar{X}_{2} C_{z x_{2}}}{\left(\bar{X}_{1}+\bar{X}_{2}\right)}+2 \frac{\bar{X}_{1} \bar{X}_{2} C_{x_{1} x_{2}}}{\left(\bar{X}_{1}+\bar{X}_{2}\right)^{4}}\right\}\right), \\
& F=\theta \bar{Z}\left(\frac{\bar{X}_{1}^{2} C_{x_{1}}^{2}}{\left(\bar{X}_{1}+\bar{X}_{2}\right)}-\bar{X}_{1} C_{z x_{1}}+\frac{\bar{X}_{1} \bar{X}_{2} C_{x_{1} x_{2}}}{\left(\bar{X}_{1}+\bar{X}_{2}\right)}\right), G=\theta \bar{Z}\left(\frac{\bar{X}_{2}^{2} C_{x_{2}}^{2}}{\left(\bar{X}_{1}+\bar{X}_{2}\right)}-\bar{X}_{2} C_{z x_{2}}+\frac{\bar{X}_{1} \bar{X}_{2} C_{x_{1} x_{2}}}{\left(\bar{X}_{1}+\bar{X}_{2}\right)}\right) .
\end{aligned}
$$

\section{THE PROPOSED SRR MODEL}

In this section, we have introduced a new SRR model by combining the three models, namely Pollock and Bek [3] additive, Hussain [9] subtractive and Eichhorn and Hayre [5] multiplicative model. The reported response $Z_{\text {new }}$ is given as,

$Z_{\text {new }}=g(Y+a S)+(1-g) Y S$,

where $g \in[0,1]$ and $\alpha \in[-1,1]$ are suitable constants controlled by the researcher. Considering $\bar{S}=E(S)=0$ and $S_{S}^{2}$ be the mean and variance of scrambling random variable $S$. The choice of ( $\mathrm{g}=1, \alpha=1)$ provides Pollock and Bek [3] additive model whereas $(\mathrm{g}=1, \alpha=-1)$, generates the subtractive model of Hussain [9]. Also, we notice that $(\mathrm{g}=0)$ will result in the multiplicative model of Eichhorn and Hayre [5], whereas for $(\mathrm{g}=1)$ we may get Tarray and Singh [18] SRR model. The resulting mean, variance and covariance for the proposed model are established as respectively

$E\left(Z_{\text {new }}\right)=g \bar{Y}$

$\operatorname{Var}\left(Z_{\text {new }}\right)=g^{2}\left(S_{y}^{2}+a^{2} S_{s}^{2}\right)+(1-g)^{2} S_{s}^{2}\left(S_{y}^{2}+\bar{Y}^{2}\right)+2 a g(1-g) \bar{Y} S_{s}^{2}$

and

$$
\sigma_{X_{i} Z_{\text {new }}}=g \sigma_{X_{i} Y}
$$

Therefore, the coefficient of variation $\left(C_{Z}\right)$ and the correlation $\left(\rho_{Z X_{1}}, \rho_{Z X 2}\right)$ will become,

$$
\begin{aligned}
& C_{Z_{\text {new }}}^{2}=\frac{1}{\bar{Z}_{\text {new }}^{2}}\left[g^{2}\left(S_{y}^{2}+a^{2} S_{s}^{2}\right)+(1-g)^{2} S_{s}^{2}\left(S_{y}^{2}+\bar{Y}^{2}\right)+2 a g(1-g) \bar{Y} S_{s}^{2}\right], \\
& \rho_{X_{i} Z_{\text {new }}}=\rho_{Y X_{i}} \frac{g S_{Y}}{\sqrt{\left[g^{2}\left(S_{y}^{2}+a^{2} S_{s}^{2}\right)+(1-g)^{2} S_{s}^{2}\left(S_{y}^{2}+\bar{Y}^{2}\right)+2 a g(1-g) \bar{Y} S_{s}^{2}\right]}},(i=1,2) .
\end{aligned}
$$

Following Gupta et al. [21] the unified measure is used to evaluate the privacy level of the proposed model and some existing models. The unified measure is given as,

$$
\delta=\frac{\operatorname{Var}\left(\hat{\mu}_{Y}\right)}{\text { Privacylevel }},
$$


where $\operatorname{Var}\left(\hat{\mu}_{Y}\right)$ is the variance of the mean estimator of the model and privacy level is the privacy measure for same model using Yan et al. [22] i-e. $E(Z-Y)^{2}$. In Table 1, the unified measures of different models are presented with the $\operatorname{Var}\left(\hat{\mu}_{Y}\right)$ and privacy level.

\section{THE PROPOSED GENERALIZED REGRESSION-CUM-EXPONENTIAL ESTIMATOR}

In this section, we have proposed an improved exponential type estimator of the finite population mean of sensitive study variable using two non-sensitive auxiliary variables. The expressions of the bias and MSE of the proposed estimator are obtained up to first order of approximation. Let $Y$ be a sensitive study variable, which cannot be directly obtained. Let $X_{1}$ and $X_{2}$ be the non-sensitive auxiliary variables, positively correlated with $Y$. Consider a scrambling variable $S$, be independent of $Y, X_{1}$ and $X_{2}$. The respondents are asked to provide true response for $X_{1}$ and $X_{2}$ and to report a scrambled response for $Y$ given as $Z=Y+S$. Suppose a random sample $n$ is drawn without replacement from a finite population $U=\left(U_{1}, U_{2}, \ldots, U_{N}\right)$. Let $Y_{i}, X_{1 i}$ and $X_{2 i}$ be the $i$ th unit values of study variable $Y$ and auxiliary variables $X_{1}$ and $X_{2}$, respectively. Let the sample means be $\bar{y}=\frac{\sum_{i=1}^{n} Y_{i}}{n}, \bar{x}_{1}=\frac{\sum_{i=1}^{n} X_{1 i}}{n}, \bar{x}_{2}=\frac{\sum_{i=1}^{n} X_{2 i}}{n}$ and $\bar{z}=\frac{\sum_{i=1}^{n} Z_{i}}{n}$ corresponding to the $\bar{Y}=E(Y), \bar{X}_{1}=E\left(X_{1}\right), \bar{X}_{2}=E\left(X_{2}\right)$ and $\bar{Z}=E(Z)$ be the population means of $Y, X_{1}, X_{2}$ and $Z$, respectively. The auxiliary variables $X_{1}$ and $X_{2}$ are assumed to be known and $\bar{S}=E(S)=0$. Therefore, $E(Z)=E(Y)$ and $C_{Z}^{2}=C_{Y}^{2}+\frac{S_{s}^{2}}{\bar{Y}^{2}}$, where $C_{Z}^{2}$ and $C_{Y}^{2}$ are the coefficient of variations of $Z$ and $Y$, respectively. 
Table 1. Unified Measure of the Different Models

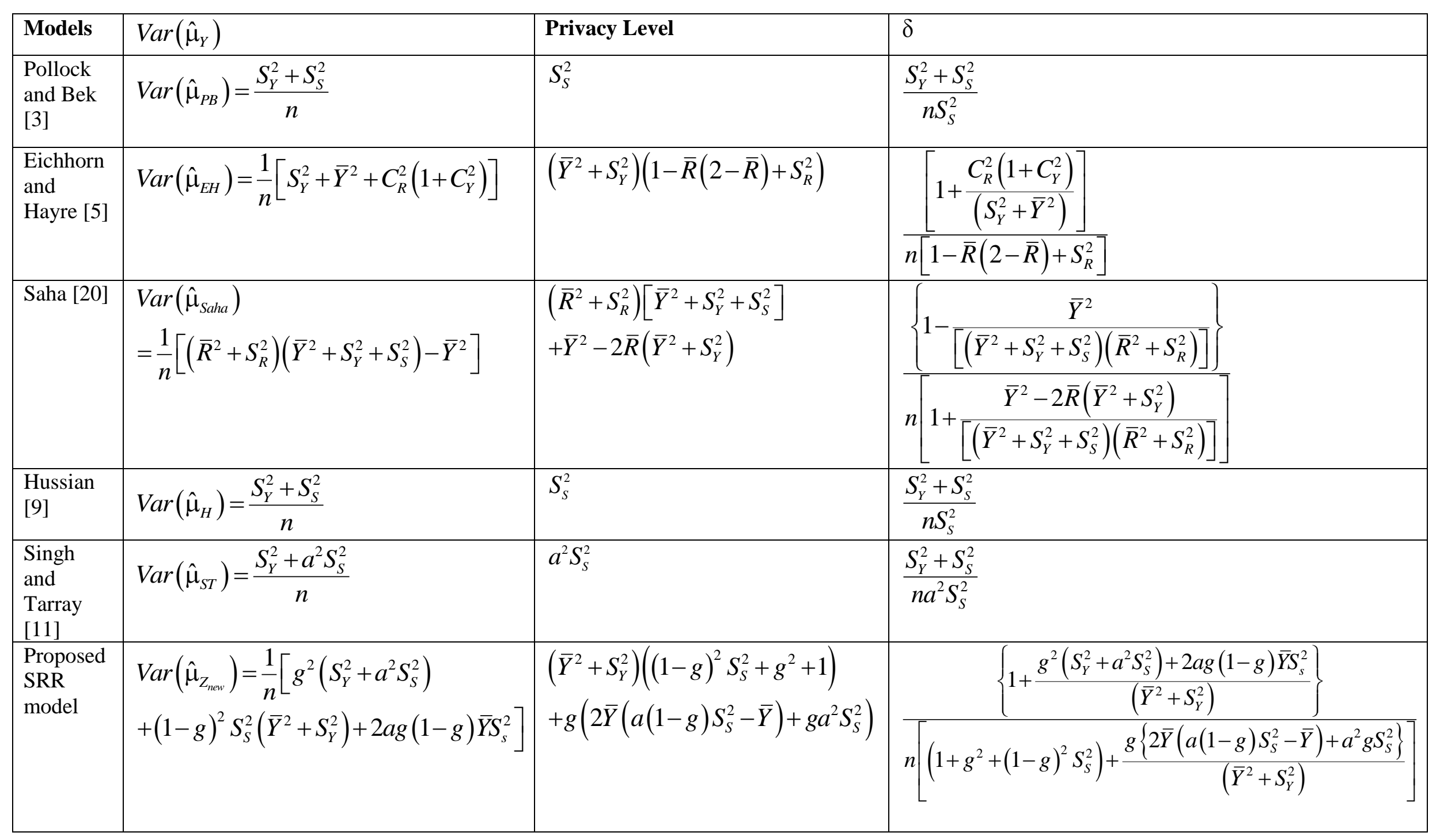


Following Rao [23] and considering a linear relation between the auxiliary variable $X_{1}$ and the sensitive study variable $Y$, we may consider a regression-type estimator $t_{\text {reg }}$ using single non-sensitive auxiliary variable under randomized response model $Z=Y+S$ as,

$t_{\text {reg }}=\alpha_{0} \bar{z}+\alpha_{1}\left(\bar{X}_{1}-\bar{x}_{1}\right)$

where $\alpha_{0}$ and $\alpha_{1}$ are the suitable constants.

Similarly following Bahl and Tuteja [24] and considering an exponential-type relation between the auxiliary variable $X_{2}$ and the sensitive study variable $Y$, we may reproduce the exponential-type ratio estimator $t_{\mathrm{e} r}$ and exponential-type product estimator $t_{\text {ep }}$ respectively under randomized response model $Z=Y+S$ as,

$t_{\mathrm{e} r}=\bar{z} \exp \left(\frac{\bar{X}_{2}-\bar{x}_{2}}{\bar{X}_{2}+\bar{x}_{2}}\right)$ and $t_{\mathrm{e} p}=\bar{z} \exp \left(\frac{\bar{x}_{2}-\bar{X}_{2}}{\bar{X}_{2}+\bar{x}_{2}}\right)$.

Then the estimators in (22)-(23) may lead us to propose a generalized regression-cum-exponential type estimator as,

$t_{g}=\left(\alpha_{0} \bar{z}+\alpha_{1}\left(\bar{X}_{1}-\bar{x}_{1}\right)\right)\left[\exp \left(\frac{b\left(\bar{X}_{2}-\bar{x}_{2}\right)}{\bar{X}_{2}+\left(\alpha_{2}-1\right) \bar{x}_{2}}\right)\right]$

where $b(-1,0,1)$ is a generalization constant its values yield regression estimator, class of regression-cumexponential ratio and regression-cum-exponential product estimator respectively. $\alpha_{0}, \alpha_{1}$ and $\alpha_{2}$ are assumed to be unknown constants and need to be estimated such that the MSE of $t_{g}$ is minimum.

It is observed that regression estimator $t_{\text {reg }}$ in (22) may be obtained for $b=0$. Also, we get exponential estimator based on single auxiliary variable. Also for different choices of these constants, we may get different estimators, as see in Table 2. It provides some example of different estimators based on two auxiliary variables.

Table 2. Class of Estimators for Two Auxiliary Variables

\begin{tabular}{lllll}
\hline Class of estimators & $\alpha_{0}$ & $\alpha_{1}$ & $b$ & $\alpha_{2}$ \\
$t_{g 1}=\left(\alpha_{0} \bar{z}+\left(\bar{X}_{1}-\bar{x}_{1}\right)\right)\left[\exp \left(\frac{\left(\bar{X}_{2}-\bar{x}_{2}\right)}{\left.\bar{X}_{2}\right)}\right)\right]$ & $\alpha_{0}$ & 1 & 1 & 1 \\
$t_{g 2}=\left(\alpha_{0} \bar{z}+b_{z x 1}\left(\bar{X}_{1}-\bar{x}_{1}\right)\right)\left[\exp \left(\frac{\left(\bar{X}_{2}-\bar{x}_{2}\right)}{\bar{X}_{2}}\right)\right]$ & $\alpha_{0}$ & $b_{z x 1}$ & 1 & 1 \\
$t_{g 3}=\left(\alpha_{0} \bar{z}+\rho_{z x 1}\left(\bar{X}_{1}-\bar{x}_{1}\right)\right)\left[\exp \left(\frac{\left(\bar{X}_{2}-\bar{x}_{2}\right)}{\bar{X}_{2}}\right)\right]$ & & & & \\
& $\alpha_{0}$ & $\rho_{z x 1}$ & 1 & 1
\end{tabular}




$$
\begin{aligned}
& t_{g 4}=\left(\alpha_{0} \bar{z}+\left(\bar{X}_{1}-\bar{x}_{1}\right)\right)\left[\exp \left(\frac{\bar{X}_{2}-\bar{x}_{2}}{2\left(\bar{X}_{2}+\bar{x}_{2}\right)}\right)\right] \\
& t_{g 5}=\left(\alpha_{0} \bar{z}+b_{z x 1}\left(\bar{X}_{1}-\bar{x}_{1}\right)\right)\left[\exp \left(\frac{\bar{X}_{2}-\bar{x}_{2}}{2 \bar{X}_{2}}\right)\right] \\
& t_{g 6}=\left(\alpha_{0} \bar{z}+C_{x 1}\left(\bar{X}_{1}-\bar{x}_{1}\right)\right)\left[\exp \left(\frac{2\left(\bar{X}_{2}-\bar{x}_{2}\right)}{\bar{X}_{2}}\right)\right] \\
& t_{g 7}=\left(\alpha_{0} \bar{z}+\rho_{z x 1}\left(\bar{X}_{1}-\bar{x}_{1}\right)\right)\left[\exp \left(\frac{\rho_{z x 2}\left(\bar{X}_{2}-\bar{x}_{2}\right)}{\bar{X}_{2}}\right)\right] \quad \begin{array}{llll}
\alpha_{0} & \rho_{z x 1} & \rho_{z x 2} & 1
\end{array} \\
& t_{g 8}=\left(\bar{z}+b_{z x 1}\left(\bar{X}_{1}-\bar{x}_{1}\right)\right)\left[\exp \left(\frac{\left(\bar{X}_{2}-\bar{x}_{2}\right)}{\bar{X}_{2}}\right)\right] \\
& t_{g 9}=\left(\bar{z}+\rho_{z x 1}\left(\bar{X}_{1}-\bar{x}_{1}\right)\right)\left[\exp \left(\frac{\left(\bar{X}_{2}-\bar{x}_{2}\right)}{\bar{X}_{2}}\right)\right] \\
& \begin{array}{llll}
\alpha_{0} & 1 & 1 / 2 & 2
\end{array} \\
& \alpha_{0} \quad b_{z x 1} \quad 1 / 2 \quad 1 \\
& \begin{array}{llll}
\alpha_{0} & C_{x 1} & 2 & 1
\end{array} \\
& 1 \quad b_{z x 1} \quad 1 \quad 1 \\
& \begin{array}{llll}
1 & \rho_{z x 1} & 1 & 1
\end{array}
\end{aligned}
$$

To obtain the bias and MSE, let us define the following notations

$e_{z}=(\bar{z}-\bar{Z}) / \bar{Z}, e_{X_{1}}=\left(\bar{X}_{1}-\bar{X}_{1}\right) / \bar{X}_{1}, e_{X_{2}}=\left(\bar{X}_{2}-\bar{X}_{2}\right) / \bar{X}_{2}$

such that $E\left(e_{i}\right)=0 \quad i=z, x_{1}, x_{2}$,

and expectations are as,

$$
\begin{aligned}
& E\left(e_{z}^{2}\right)=\theta C_{z}^{2}, E\left(e_{x_{1}}^{2}\right)=\theta C_{x_{1}}^{2}, E\left(e_{x_{2}}^{2}\right)=\theta C_{x_{2}}^{2}, E\left(e_{z} e_{x_{1}}\right)=\theta C_{z} C_{x_{1}} \rho_{z x_{1}}, \\
& E\left(e_{z} e_{x_{2}}\right)=\theta C_{z} C_{x_{2}} \rho_{z x_{2}}, E\left(e_{x_{1}} e_{x_{2}}\right)=\theta C_{x_{1}} C_{x_{2}} \rho_{x_{1} x_{2}}
\end{aligned}
$$

where $\theta=(1-f) / n, f=n / N$. We may express (24) in terms of $e$ 's as,

$$
\begin{aligned}
t_{g} & \cong\left(\alpha_{0} \bar{Z}\left(1+e_{Z}\right)-\alpha_{1} \bar{X}_{1} e_{X_{1}}\right) \exp \left(\frac{-b e_{X_{2}}}{\alpha_{2}}\left(1+\left(1-\frac{1}{\alpha_{2}}\right) e_{X_{2}}\right)^{-1}\right) \\
& =\left(\alpha_{0} \bar{Z}\left(1+e_{Z}\right)-\alpha_{1} \bar{X}_{1} e_{X_{1}}\right) \exp \left(\frac{-b e_{X_{2}}}{\alpha_{2}}\left(1-\left(1-\frac{1}{\alpha_{2}}\right) e_{X_{2}}+\ldots\right)\right) .
\end{aligned}
$$

The bias and MSE of $t_{g}$ to the first order approximation $O\left(\mathrm{n}^{-1}\right)$ are given by,

$$
\operatorname{Bias}\left(t_{g}\right) \cong \bar{Z}\left[\alpha_{0}\left(1+\frac{b^{2}}{\alpha_{2}^{2}} \theta C_{X_{2}}^{2}-\frac{b}{\alpha_{2}} \theta C_{Z} C_{X_{2}} \rho_{Z X_{2}}\right)+\alpha_{1} \frac{\bar{X}_{1}}{\bar{Z}} \frac{b}{\alpha_{2}} \theta C_{X_{1}} C_{X_{2}} \rho_{X_{1} X_{2}}-1\right]
$$


Equating (27) with zero, we may obtain

$$
\alpha_{2}=\left(\frac{2 \alpha_{0} b}{A_{1}-\sqrt{A_{1}^{2}-4 A_{2}}}, \frac{2 \alpha_{0} b}{A_{1}+\sqrt{A_{1}^{2}-4 A_{2}}}\right) \text {, }
$$

where

$$
A_{1}=\left(\alpha_{0} K_{Z X_{2}}-\alpha_{1} \frac{K_{X_{1} X_{2}}}{R_{Z X_{1}}}\right), A_{2}=\frac{\alpha_{0}\left(\alpha_{0}-1\right)}{\theta C_{X_{2}}^{2}}, K_{Z X_{2}}=\frac{C_{Z}}{C_{X_{2}}} \rho_{Z X_{2}}, K_{X_{1} X_{2}}=\frac{C_{X_{1}}}{C_{X_{2}}} \rho_{X_{1} X_{2}}, R_{Z X_{1}}=\frac{\bar{Z}}{\bar{X}_{1}} .
$$

By using the values of $\alpha_{2}$, one may get an almost unbiased class of estimators for the population mean of sensitive study variable. Providing different values of the constants to (27), we can get expressions for the bias of different estimators.

A general MSE expression of the $t_{g}$ can be obtained as,

$$
\begin{aligned}
\operatorname{MSE}\left(t_{g i}\right) & \cong \theta \bar{Z}^{2}\left[\left\{\alpha_{0 i}^{2}\left(1+C_{Z}^{2}\right)+\alpha^{2}{ }_{1 i} \frac{\bar{X}_{1}^{2}}{\bar{Z}^{2}} C_{X_{1}}^{2}+\alpha_{0 i}^{2} v_{i}^{2} C_{X_{2}}^{2}-2 \alpha_{0 i} \alpha_{1 i} \frac{\bar{X}_{1}}{\bar{Z}} C_{Z} C_{X_{2}} \rho_{Z X_{1}}\right.\right. \\
& \left.\left.-2 \alpha_{0 i}^{2} \nu_{i} C_{Z} C_{X_{2}} \rho_{Z X_{2}}+2 \alpha_{0 i}^{2} \alpha_{1 i} \frac{\bar{X}_{1}}{\bar{Z}} v_{i} C_{X_{1}} C_{X_{2}} \rho_{X_{1} X_{2}}\right\}+1-2 \alpha_{0 i}\right],
\end{aligned}
$$

where $v=\frac{b}{\alpha_{2}}$ and $\alpha_{0}, \alpha_{1}$ and $v$ attain their optimum values as,

$\alpha_{0}=\frac{1}{\left(1+C_{Z} R_{3}\right)}, \alpha_{1}=R_{1} \frac{\bar{Z} C_{Z}}{\bar{X} C_{x_{1}}\left(1+C_{Z} R_{3}\right)}, v=R_{2} \frac{C_{Z}}{C_{X_{2}}}$

where

$R_{1}=\frac{\left(\rho_{x 1 z}-\rho_{x 2 z} \rho_{x 1 x 2}\right)}{\left(1-\rho_{x 1 x 2}^{2}\right)}, R_{2}=\frac{\left(\rho_{z x 2}-\rho_{z x 1} \rho_{x 1 x 2}\right)}{\left(1-\rho_{x 1 x 2}^{2}\right)}$

and $R_{3}=R_{1}^{2}+R_{2}^{2}-2 R_{1} \rho_{z x 1}-2 R_{2} \rho_{z x 2}+2 R_{1} R_{2} \rho_{x 1 x 2}$.

Substituting the optimum values in (30), we may get the minimum value of the $\operatorname{MSE}\left(t_{g}\right)$ as,

$$
\min \operatorname{MSE}\left(t_{g}\right)=\theta \bar{Z}^{2} C^{2}{ }_{Z}\left[1-\frac{1}{\left(1+R_{3} C_{z}^{2}\right)}\right] .
$$

We may get the minimum MSEs for $t_{g i}(i=1,2, \ldots, 9)$ using different values of $\alpha_{0}, \alpha_{1}, \alpha_{2}$ and $b$ in (32), (e.g. as given in Table 2).

Observe that optimum choices of the constants $\alpha_{0}, \alpha_{1}$ and $\alpha_{2}$ are based on some parameters whose values may be from the prior surveys or may be presumed through the experience drawn in due course of time, for case in point see, Horvitz and Thompson [25], Sanaullah et al. [26], Asghar et al. [27], and Sanaullah et al. [28]. In many real life situations, it may not be possible to guess these values through experience or prior surveys, so it is better to replace these values with the estimates drawn from a pilot surveys for case in point 
see Koyuncu and Kadilar [29] and Jabeen et al. [30]. Also, we can verify that the generalized regressioncum-exponential estimator $t_{g}$ in (24) is more efficient than the estimators $t_{m}, t_{S}$ and $t_{G}$ when

$$
\begin{aligned}
& {\left[S_{S}^{2}+\frac{S_{Y}^{2}}{\left(1+R_{3} C_{Z}^{2}\right)}\right]>0,\left[C_{X 1}^{2}-2 C_{Z X 1}+\frac{C_{Z}^{2}}{\left(1+R_{3} C_{Z}^{2}\right)}\right]>0 \text { and }} \\
& {\left[\frac{\left(1-\rho_{Z X 1}^{2}\right)\left(1+\theta C_{X}^{2}\right)}{C_{Z}^{2}\left(1-\rho_{Z X 1}^{2}\right) \theta+\left(1+\theta C_{X}^{2}\right)}-\frac{R_{3} C_{Z}^{2}}{\left(1+R_{3} C_{Z}^{2}\right)}\right]>0 \text {, respectively. }}
\end{aligned}
$$

Eq. (27)-(32) can be reproduced under the proposed model following the results in Eq. (15)-(20) for the proposed estimator as well as for the existing estimators.

\section{SIMULATION STUDY}

In this section the efficiency of the proposed class of estimators over existing estimators using proposed model and Pollock and Bek [3] is provided using real data and with a simulation study. This study is involved to evaluate the MSEs of the estimators both empirically and theoretically. This simulation study considers two finite populations of size $\mathrm{N}=1000$ each generated from multivariate normal distribution with theoretical mean vector $\mu=[5,5,5]$ for $\left[\mathrm{Y}, \mathrm{X}_{1}, \mathrm{X}_{2}\right]$ with covariance matrices for two populations respectively as,

\section{Population I}

$$
\sigma^{2}=\left[\begin{array}{ccc}
10 & 3 & 2.9 \\
3 & 2 & 1.1 \\
2.9 & 1.1 & 2
\end{array}\right] \quad \rho_{x 1 y}=0.6817, \rho_{x 2 y}=0.6705
$$

\section{Population II}

$$
\sigma^{2}=\left[\begin{array}{ccc}
6 & 3 & 2.9 \\
3 & 2 & 1.1 \\
2.9 & 1.1 & 2
\end{array}\right] \quad \rho_{x 1 y}=0.8706, \rho_{x 2 y}=0.8428
$$

The scrambling variable $S$ is taken from the normal distribution with mean zero and standard deviation equal to $10 \%$ of the standard deviation of $\mathrm{X}_{1}$.

The reported response for the two models are taken as,

i) Pollock and Bek [3] $\rightarrow \quad Z=Y+S$

ii) New model

a) $Z=0.3(Y+0.5 S)+(1-0.3) Y S$ iff $g=0.3 \& \mathrm{a}=0.5$

b) $Z=0.6(Y+0.5 S)+(1-0.6) Y S$ iff $g=0.6 \& \mathrm{a}=0.5$

c) $Z=0.9(Y+0.5 S)+(1-0.9) Y S$ iff $\mathrm{g}=0.9 \& \mathrm{a}=0.5$ 
The sample size for each population considered as $n=50,100,200$ and 300. The percent relative efficiency (PRE)s for Himmelfarb and Edgel [4] model and new model are calculated with the following equations:

$\operatorname{PRE}=\frac{\operatorname{MSE}\left(t_{m}\right)}{\operatorname{MSE}\left(t_{j}\right)} \times 100$,

where $j=m, S, G, K_{1}, K_{2}, g$ and $g_{i}(\mathrm{i}=1,2, \ldots, 9)$.

In Table 3, the simulation results of the unified measure for different models are presented under both populations.

Table 3. Simulation results for unified measure

\begin{tabular}{|l|l|l|l|}
\hline $\mathbf{n}$ & SRR Model & \multicolumn{2}{|l|}{ Unified Measure } \\
\cline { 3 - 4 } & & Population I & Population II \\
\hline 50 & Pollock and Bek [3] & 9.85535 & 7.47312 \\
\cline { 2 - 4 } & Eichhorn and Hayre [5] & 0.02042 & 0.02042 \\
\cline { 2 - 4 } & Saha [20] & 0.00342 & 0.00235 \\
\cline { 2 - 4 } & Hussian [9] & 9.85535 & 7.47312 \\
\cline { 2 - 4 } & Singh and Tarray [11] & 39.4214 & 29.8925 \\
\cline { 2 - 4 } & Proposed SRR model & $\mathbf{0 . 0 0 3 0 8}$ & $\mathbf{0 . 0 0 2 2 7}$ \\
\hline 100 & Pollock and Bek [3] & 4.92767 & 3.09094 \\
\cline { 2 - 4 } & Eichhorn and Hayre [5] & 0.01010 & 0.01010 \\
\cline { 2 - 4 } & Saha [20] & 0.00171 & 0.00119 \\
\cline { 2 - 4 } & Hussian [9] & 4.92767 & 3.09094 \\
\cline { 2 - 4 } & Singh and Tarray [11] & 19.7107 & 12.3637 \\
\cline { 2 - 4 } & Proposed SRR model & $\mathbf{0 . 0 0 1 5 4}$ & $\mathbf{0 . 0 0 1 1 2}$ \\
\hline \multirow{5}{*}{200} & Pollock and Bek [3] & 2.61486 & 1.54547 \\
\cline { 2 - 4 } & Eichhorn and Hayre [5] & 0.00502 & 0.00502 \\
\cline { 2 - 4 } & Saha [20] & 0.00091 & 0.00059 \\
\cline { 2 - 4 } & Hussian [9] & 2.61486 & 1.54547 \\
\cline { 2 - 4 } & Singh and Tarray [11] & 10.4594 & 6.18188 \\
\cline { 2 - 4 } & Proposed SRR model & $\mathbf{0 . 0 0 0 8 5}$ & $\mathbf{0 . 0 0 0 5 6}$ \\
\hline \multirow{5}{*}{300} & Pollock and Bek [3] & 1.74324 & 1.03031 \\
\cline { 2 - 4 } & Eichhorn and Hayre [5] & 0.00334 & 0.00334 \\
\cline { 2 - 4 } & Saha [20] & 0.00061 & 0.00039 \\
\cline { 2 - 4 } & Hussian [9] & 1.74324 & 1.03031 \\
\cline { 2 - 4 } & Singh and Tarray [11] & $\mathbf{0 . 0 0 0 5 7}$ & $\mathbf{0 . 0 0 0 3 7}$ \\
\cline { 2 - 4 } & Proposed SRR model & & \\
\hline
\end{tabular}

Using the two models, the proposed SRR model and Pollock and Bek's model the PRE values for each estimator are shown in Tables 4-11.

\section{CONCLUSION}

This paper has proposed a new SRR model and a generalized regression-cum-exponential estimator using two non-sensitive auxiliary variables to obtain efficient results for estimating the population mean of sensitive variable and some class of estimators have also been shown in Table2. The unified measure is calculated for different models presented in Table 1.The unified measure of the proposed SRR model has 
been obtained using $g=0.6$. From Table 3 , it has been noticed that the proposed model has minimum value unified measure as compared to existing models. As the unified measure decreases the privacy of the respondent increases.

Also, from the simulation and empirical study using two population datasets provided in Table (4-11), it has been observed that the MSE values for the proposed class of estimators are less than the MSEs of the existing estimators $t_{m}, t_{S}, t_{G}, t_{K_{1}}, t_{K_{2}}, t_{r e g}$, and $t_{e r}$ when both models are used. It shows that the class of generalized estimators are more efficient than existing estimators. From both populations, we have noticed that as the sample size increases, there is a reduction in the $M S E$ of the estimators discussed.

From Table (4-11) it is observed that using the proposed SRR model the generalized class of estimators including the existing estimators attains less MSE values than the MSE value using the Pollock and Bek's model therefore it can be concluded that the proposed SRR model improves the efficiency of the proposed class of estimators and the efficiency of the existing estimators too. Furthermore, we observed what can be noted from Table (4-11) is that as the value of $g$ increases in the proposed SRR model, the MSE's of estimators also increases. From both populations, we have noticed that as the sample size increases, there is a reduction in the $M S E$ of the estimators discussed. The same type of results is noted in Diana and Perri [8] model.

\section{CONFLICTS OF INTEREST}

No conflict of interest was declared by the authors.

\section{REFERENCES}

[1] Warner, S. L., "Randomized response: A survey technique for eliminating evasive answer bias", Journal of the American Statistical Association, 60(309): 63-69, (1965).

[2] Greenberg, B. G., Kuebler, R. R., Jr., Abernathy, J. R., Horvitz, D. G., “Application of the randomized response technique in obtaining quantitative data", Journal of the American Statistical Association, 66(334): 243-250, (1971).

[3] Pollock, K. H. and Bek, Y., "A comparison of three randomized response models for quantitative data”, Journal of the American Statistical Association, 71(356):884-886, (1976).

[4] Himmelfarb, S., Edgell, S. E., "Additive constants model: A randomized response technique for eliminating evasiveness to quantitative response questions", Psychological Bulletin, 87(3): 525, (1980).

[5] Eichhorn, B.H., Hayre, L. S., "Scrambled randomized response models for obtaining sensitive quantitative data", Journal of Statistical Planning and Inference, 7(4): 307-316, (1983).

[6] Saha, A., "Optional randomized response in stratified unequal probability sampling-A simulation based numerical study with Kuk’s method", Test, 16(2): 346-354, (2007).

[7] Diana, G., Perri, P. F., "New scrambled response models for estimating the mean of a sensitive quantitative character", Journal of Applied Statistics, 37(11): 1875-1890, (2010).

[8] Diana, G., Perri, P. F., “A class of estimators for quantitative sensitive data”, Statistical Papers, 52(3): 633-650, (2011). 
[9] Hussain, Z., "Improvement of the Gupta and Thornton scrambling model through double use of randomization device", International Journal Academic Research Business and Social Science, 2:9197, (2012).

[10] Chen, C. C., Singh, S., "The Franklin's randomized response model for two sensitive attributes", Section Survey Research Methods, 4171-4185, (2009).

[11] Singh, H., Tarray, T., "An improved randomized response additive model”, Sri Lankan Journal of Applied Statistics, 15(2): 131-138, (2014).

[12] Hussain, Z., Al-Zahrani, B., "Mean and sensitivity estimation of a sensitive variable through additive scrambling", Communications in Statistics-Theory and Methods, 45(1): 182-193, (2016).

[13] Bouza-Herrera, C. N., "Behavior of some scrambled randomized response models under simple random sampling, ranked set sampling and Rao-Hartley-Cochran designs", Handbook of Statistics, 34, 209220, Elsevier, (2016).

[14] Bouza-Herrera, C. N., "Data gathering, analysis and protection of privacy through randomized response techniques: qualitative and quantitative human traits", Investigación Operacional, 39(1): 151-152, (2018).

[15] Bouza, C. N., Singh, P., Singh, R., "Ranked set sampling and optional scrambling randomized response modeling”, Investigación Operacional, 39(1): 100-107, (2018).

[16] Sousa, R., Shabbir, J., Real, P. C., Gupta, S., "Ratio estimation of the mean of a sensitive variable in the presence of auxiliary information", Journal of Statistical Theory and Practice, 4(3): 495-507, (2010).

[17] Gupta, S., Shabbir, J., Sousa, R., Corte-real, P., "Estimation of the mean of a sensitive variable in the presence of auxiliary information", Communications in Statistics-Theory and Methods, 41(13-14): 2394-2404, (2012).

[18] Tarray, T. A., Singh, H. P., “A general procedure for estimating the mean of a sensitive variable using auxiliary information", Investigacion Operacionel, 36(3): 268-279, (2015).

[19] Koyuncu, N., Gupta, S., Sousa, R., "Exponential-type estimators of the mean of a sensitive variable in the presence of non-sensitive auxiliary information", Communications in Statistics-Simulation and Computation, 43(7): 1583-1594, (2014).

[20] Saha, A., "A randomized response technique for quantitative data under unequal probability sampling", Journal of Statistical Theory and Practice, 2(4): 589-596, (2008).

[21] Gupta, S., Mehta, S., Shabbir, J., Khalil, S., "A unified measure of respondent privacy and model efficiency in quantitative RRT models", Journal of Statistical Theory and Practice, 12(3): 506-511, (2018).

[22] Yan, Z., Wang, J., Lai, J., "An efficiency and protection degree-based comparison among the quantitative randomized response strategies", Communications in Statistics-Theory and Methods, 38(3): 400-408, (2008). 
[23] Rao, T. J., "On certail methods of improving ration and regression estimators", Communications in Statistics-Theory and Methods, 20(10): 3325-3340, (1991).

[24] Bahl, S., Tuteja, R. K., "Ratio and product type exponential estimators", Journal of Information and Optimization Sciences”, 12(1): 159-164, (1991).

[25] Horvitz, D.G., Thompson, D.J., "A generalization of sampling without replacement from a finite universe", J. Amer. Statist. Assoc., 47(260): 663-685, (1952).

[26] Sanaullah, A., Ali, H.A., Noor ul Amin, M., Hanif, M., "Generalized exponential chain ratio estimators under stratified two-phase random sampling", Applied Mathematics and Computation, 226: 541-547, (2014).

[27] Asghar, A., Sanaullah, A., Hanif, M., "Generalized exponential type estimator for population variance in survey sampling”, Revista Colombiana de Estadística, 37(1): 213-224, (2014).

[28] Sanaullah, A., Hanif, M., Asghar. A., "Generalized exponential estimators for population variance under two-phase sampling", Int. J. Appl. Comput. Math., 2(1): 75-84, (2016).

[29] Koyuncu, N., Kadilar, C., "Family of estimators of population mean using two auxiliary variables in stratified random sampling", Communications in Statistics-Theory and Methods, 38(14): 2398-2417, (2009).

[30] Jabeen, R., Sanaullah, A., Hanif, M., "Generalized estimator for estimating population mean under two stage sampling”, Pak. J. Statist., 30(4): 465-486, (2014). 
Table 4. Simulation and empirical results at sample size $n=50$ for the MSEs and PREs of the estimators using population I $\left[N=1000, \rho_{x 1 y}=0.6817 \& \rho_{x 2 y}=0.6705\right]$

\begin{tabular}{|c|c|c|c|c|c|c|c|c|c|c|c|c|}
\hline \multirow[b]{4}{*}{ Estimators } & \multicolumn{12}{|c|}{ MSE Estimation using Population-I } \\
\hline & \multirow{2}{*}{\multicolumn{3}{|c|}{ Himmelfarb and Edgel Model }} & \multicolumn{9}{|c|}{ Proposed Model } \\
\hline & & & & \multicolumn{3}{|c|}{$\mathrm{g}=0.3 \& \mathrm{a}=0.5$} & \multicolumn{3}{|c|}{$\mathrm{g}=0.6 \& \mathrm{a}=0.5$} & \multicolumn{3}{|c|}{$\mathrm{g}=0.9 \& \mathrm{a}=0.5$} \\
\hline & Empirical & Theoretical & PRE & Empirical & Theoretical & PRE & Empirical & Theoretical & PRE & Empirical & Theoretical & PRE \\
\hline$t_{m}$ & 0.2148 & 0.1904 & 100.00 & 0.0180 & 0.0199 & 100.00 & 0.0208 & 0.0693 & 100.00 & 0.0233 & 0.1540 & 100.00 \\
\hline$t_{S}$ & 0.1168 & 0.1125 & 169.18 & 0.0174 & 0.0166 & 119.74 & 0.0228 & 0.0425 & 163.00 & 0.0339 & 0.0911 & 169.17 \\
\hline$t_{G}$ & 0.0924 & 0.1017 & 187.25 & 0.0157 & 0.0156 & 127.81 & 0.0167 & 0.0386 & 179.59 & 0.0202 & 0.0823 & 187.27 \\
\hline$t_{K_{2}}$ & 3.4328 & 0.4666 & 40.80 & 0.1195 & 0.0486 & 40.91 & 0.1909 & 0.1713 & 40.48 & 0.3578 & 0.3809 & 40.44 \\
\hline$t_{1}$ & 0.0924 & 0.1017 & 187.27 & 0.0157 & 0.0156 & 127.81 & 0.0167 & 0.0386 & 179.59 & 0.0202 & 0.0823 & 187.27 \\
\hline$t_{2}$ & 0.1619 & 0.1429 & 133.22 & 0.0175 & 0.0194 & 102.84 & 0.0178 & 0.0535 & 129.68 & 0.0264 & 0.1157 & 133.17 \\
\hline \multicolumn{13}{|l|}{ Proposed } \\
\hline$t_{g}$ & 0.0729 & 0.0780 & 243.95* & 0.0157 & 0.0135 & $147.85 *$ & 0.0167 & 0.0301 & $230.37 *$ & 0.0202 & 0.0631 & 244.03* \\
\hline$t_{g 5}$ & 0.0796 & 0.0863 & 220.68 & 0.0193 & 0.0167 & 119.23 & 0.0308 & 0.0431 & 160.99 & 0.0341 & 0.0923 & 166.88 \\
\hline$t_{g 6}$ & 0.1097 & 0.0962 & 198.00 & 0.0363 & 0.0170 & 117.13 & 0.1161 & 0.0406 & 170.66 & 0.1381 & 0.0871 & 176.88 \\
\hline$t_{g 7}$ & 0.0890 & 0.0979 & 194.56 & 0.0172 & 0.0315 & 63.15 & 0.0210 & 0.0663 & 104.60 & 0.0267 & 0.1232 & 125.05 \\
\hline$t_{g 8}$ & 0.0862 & 0.0897 & 212.19 & 0.0233 & 0.0144 & 137.91 & 0.0511 & 0.0340 & 204.18 & 0.0545 & 0.0718 & 214.62 \\
\hline$t_{g 9}$ & 0.0809 & 0.0813 & 234.14 & 0.0223 & 0.0166 & 119.59 & 0.0465 & 0.0304 & 227.87 & 0.0533 & 0.0646 & 238.47 \\
\hline
\end{tabular}

*shows that most efficient estimator under each model 
Table 5. Simulation and empirical results at sample size $n=100$ for the MSEs and PREs of the estimators using population I $\left[N=1000, \rho_{x 1 y}=0.6817 \& \rho_{x 2 y}=0.6705\right]$

\begin{tabular}{|c|c|c|c|c|c|c|c|c|c|c|c|c|}
\hline \multirow{4}{*}{$\begin{array}{l}\mathrm{n}=100 \\
\text { Estimators }\end{array}$} & \multicolumn{12}{|c|}{ MSE Estimation using Population-I } \\
\hline & \multirow{2}{*}{\multicolumn{3}{|c|}{ Himmelfarb and Edgel Model }} & \multicolumn{9}{|c|}{ Proposed Model } \\
\hline & & & & \multicolumn{3}{|c|}{$\mathrm{g}=0.3 \& \mathrm{a}=0.5$} & \multicolumn{3}{|c|}{$g=0.6 \& a=0.5$} & \multicolumn{3}{|c|}{$\mathrm{g}=0.9 \& \mathrm{a}=0.5$} \\
\hline & Empirical & Theoretical & PRE & Empirical & Theoretical & PRE & Empirical & Theoretical & PRE & Empirical & Theoretical & PRE \\
\hline$t_{m}$ & 0.1010 & 0.0902 & 100.00 & 0.0056 & 0.0094 & 100.00 & 0.0065 & 0.0329 & 100.00 & 0.0107 & 0.0730 & 100.00 \\
\hline$t_{S}$ & 0.0574 & 0.0533 & 169.19 & 0.0054 & 0.0079 & 119.82 & 0.0089 & 0.0202 & 163.03 & 0.0186 & 0.0431 & 169.16 \\
\hline$t_{G}$ & 0.0504 & 0.0483 & 186.86 & 0.0051 & 0.0074 & 127.43 & 0.0059 & 0.0183 & 179.21 & 0.0102 & 0.0391 & 186.84 \\
\hline$t_{K_{1}}$ & 0.0504 & 0.0483 & 186.90 & 0.0051 & 0.0073 & 128.65 & 0.0059 & 0.0183 & 179.61 & 0.0102 & 0.0390 & 186.89 \\
\hline$t_{K_{2}}$ & 2.4119 & 0.2223 & 40.56 & 0.0488 & 0.0390 & 24.19 & 0.2220 & 0.1449 & 22.68 & 0.7442 & 0.3238 & 22.54 \\
\hline$t_{1}$ & 0.0504 & 0.0483 & 186.86 & 0.0051 & 0.0074 & 127.43 & 0.0059 & 0.0183 & 179.21 & 0.0102 & 0.0391 & 186.84 \\
\hline$t_{2}$ & 0.0775 & 0.0677 & 133.22 & 0.0056 & 0.0092 & 102.84 & 0.0071 & 0.0253 & 129.69 & 0.0124 & 0.0548 & 133.19 \\
\hline \multicolumn{13}{|l|}{ Proposed } \\
\hline$t_{g}$ & 0.0389 & 0.0370 & 243.53* & 0.0051 & 0.0064 & $147.57 *$ & 0.0059 & 0.0143 & 230.04* & 0.0102 & 0.0300 & 243.61* \\
\hline$t_{g_{1}}$ & 0.0390 & 0.0371 & 243.20 & 0.0252 & 0.0154 & 61.11 & 0.0305 & 0.0174 & 188.58 & 0.0424 & 0.0303 & 241.19 \\
\hline$t_{g 2}$ & 0.0441 & 0.0425 & 212.04 & 0.0087 & 0.0069 & 137.66 & 0.0117 & 0.0161 & 204.04 & 0.0254 & 0.0340 & 214.46 \\
\hline$t_{g 3}$ & 0.0418 & 0.0386 & 233.63 & 0.0082 & 0.0079 & 119.52 & 0.0127 & 0.0144 & 227.49 & 0.0225 & 0.0307 & 238.04 \\
\hline$t_{g 4}$ & 0.0494 & 0.0458 & 197.03 & 0.0193 & 0.0131 & 71.98 & 0.0195 & 0.0170 & 193.35 & 0.0239 & 0.0360 & 202.84 \\
\hline$t_{g 5}$ & 0.0429 & 0.0409 & 220.44 & 0.0068 & 0.0079 & 118.92 & 0.0074 & 0.0204 & 160.71 & 0.0150 & 0.0438 & 166.58 \\
\hline$t_{g 6}$ & 0.0544 & 0.0457 & 197.55 & 0.0138 & 0.0081 & 116.71 & 0.0299 & 0.0193 & 170.30 & 0.0621 & 0.0414 & 176.44 \\
\hline$t_{g 7}$ & 0.0459 & 0.0465 & 194.10 & 0.0056 & 0.0150 & 62.99 & 0.0064 & 0.0315 & 104.39 & 0.0104 & 0.0585 & 124.76 \\
\hline$t_{g 8}$ & 0.0441 & 0.0425 & 212.04 & 0.0087 & 0.0069 & 137.66 & 0.0117 & 0.0161 & 204.04 & 0.0254 & 0.0340 & 214.46 \\
\hline$t_{g_{9}}$ & 0.0418 & 0.0386 & 233.63 & 0.0082 & 0.0079 & 119.52 & 0.0127 & 0.0144 & 227.49 & 0.0225 & 0.0307 & 238.04 \\
\hline
\end{tabular}


Table 6. Simulation and empirical results at sample size $n=200$ for the MSEs and PREs of the estimators using population I $\left[N=1000, \rho_{x 1 y}=0.6817 \& \rho_{x 2 y}=0.6705\right]$

\begin{tabular}{|c|c|c|c|c|c|c|c|c|c|c|c|c|}
\hline \multirow{4}{*}{$\begin{array}{l}\mathrm{n}=200 \\
\text { Estimators }\end{array}$} & \multicolumn{12}{|c|}{ MSE Estimation using Population-I } \\
\hline & \multirow{2}{*}{\multicolumn{3}{|c|}{ Himmelfarb and Edgel Model }} & \multicolumn{9}{|c|}{ Proposed Model } \\
\hline & & & & \multicolumn{3}{|c|}{$\mathrm{g}=0.3 \& \mathrm{a}=0.5$} & \multicolumn{3}{|c|}{$\mathrm{g}=0.6 \& \mathrm{a}=0.5$} & \multicolumn{3}{|c|}{$\mathrm{g}=0.9 \& \mathrm{a}=0.5$} \\
\hline & Empirical & Theoretical & PRE & Empirical & Theoretical & PRE & Empirical & Theoretical & PRE & Empirical & Theoretical & PRE \\
\hline$t_{m}$ & 0.0399 & 0.0401 & 100.00 & 0.0030 & 0.0042 & 100.00 & 0.0033 & 0.0146 & 100.00 & 0.0039 & 0.0324 & 100.00 \\
\hline$t_{S}$ & 0.0241 & 0.0237 & 169.19 & 0.0029 & 0.0035 & 119.71 & 0.0045 & 0.0090 & 162.95 & 0.0075 & 0.0192 & 169.17 \\
\hline$t_{G}$ & 0.0227 & 0.0215 & 186.68 & 0.0028 & 0.0033 & 126.97 & 0.0031 & 0.0082 & 178.92 & 0.0037 & 0.0174 & 186.70 \\
\hline$t_{K_{1}}$ & 0.0226 & 0.0214 & 187.46 & 0.0027 & 0.0033 & 128.92 & 0.0030 & 0.0081 & 180.47 & 0.0036 & 0.0174 & 186.81 \\
\hline$t_{K_{2}}$ & 1.7998 & 0.0991 & 40.45 & 0.0965 & 0.0341 & 12.28 & 0.2769 & 0.1315 & 11.10 & 0.8645 & 0.2950 & 10.99 \\
\hline$t_{1}$ & 0.0227 & 0.0215 & 186.68 & 0.0028 & 0.0033 & 126.97 & 0.0031 & 0.0082 & 178.92 & 0.0037 & 0.0174 & 186.70 \\
\hline$t_{2}$ & 0.0301 & 0.0301 & 133.20 & 0.0030 & 0.0041 & 102.95 & 0.0036 & 0.0113 & 129.66 & 0.0050 & 0.0244 & 133.18 \\
\hline \multicolumn{13}{|l|}{ Proposed } \\
\hline$t_{g}$ & 0.0178 & 0.0165 & 243.35* & 0.0028 & 0.0029 & $147.02 *$ & 0.0031 & 0.0064 & 229.56* & 0.0037 & $\mathbf{0 . 0 1 3 3}$ & 243.47* \\
\hline$t_{g_{1}}$ & 0.0179 & 0.0165 & 243.06 & 0.0114 & 0.0069 & 61.08 & 0.0149 & 0.0077 & 188.63 & 0.0211 & 0.0135 & 240.94 \\
\hline$t_{g 2}$ & 0.0203 & 0.0189 & 211.95 & 0.0040 & 0.0031 & 137.38 & 0.0063 & 0.0072 & 203.91 & 0.0119 & 0.0151 & 214.34 \\
\hline$t_{g 3}$ & 0.0181 & 0.0172 & 233.43 & 0.0042 & 0.0035 & 119.37 & 0.0062 & 0.0064 & 227.41 & 0.0111 & 0.0136 & 237.76 \\
\hline$t_{g 4}$ & 0.0212 & 0.0204 & 196.76 & 0.0086 & 0.0058 & 71.99 & 0.0093 & 0.0076 & 193.12 & 0.0104 & 0.0160 & 202.56 \\
\hline$t_{g 5}$ & 0.0196 & 0.0182 & 220.22 & 0.0032 & 0.0035 & 118.70 & 0.0040 & 0.0091 & 160.62 & 0.0061 & 0.0195 & 166.39 \\
\hline$t_{g 6}$ & 0.0223 & 0.0203 & 197.34 & 0.0071 & 0.0036 & 116.39 & 0.0153 & 0.0086 & 170.16 & 0.0331 & 0.0184 & 176.25 \\
\hline$t_{g 7}$ & 0.0196 & 0.0207 & 193.90 & 0.0030 & 0.0067 & 62.91 & 0.0032 & 0.0140 & 104.29 & 0.0038 & 0.0260 & 124.63 \\
\hline$t_{g 8}$ & 0.0203 & 0.0189 & 211.95 & 0.0040 & 0.0031 & 137.38 & 0.0063 & 0.0072 & 203.91 & 0.0119 & 0.0151 & 214.34 \\
\hline$t_{g_{9}}$ & 0.0181 & 0.0172 & 233.43 & 0.0042 & 0.0035 & 119.37 & 0.0062 & 0.0064 & 227.41 & 0.0111 & 0.0136 & 237.76 \\
\hline
\end{tabular}


Table 7. Simulation and empirical results at sample size $n=300$ for the MSEs and PREs of the estimators using population I $\left[N=1000, \rho_{x 1 y}=0.6817 \& \rho_{x 2 y}=0.6705\right]$

\begin{tabular}{|c|c|c|c|c|c|c|c|c|c|c|c|c|}
\hline \multirow{4}{*}{$\begin{array}{l}\mathrm{n}=\mathbf{3 0 0} \\
\text { Estimators }\end{array}$} & \multicolumn{12}{|c|}{ MSE Estimation using Population-I } \\
\hline & \multirow{2}{*}{\multicolumn{3}{|c|}{ Himmelfarb and Edgel Model }} & \multicolumn{9}{|c|}{ Proposed Model } \\
\hline & & & & \multicolumn{3}{|c|}{$\mathrm{g}=0.3 \& \mathrm{a}=0.5$} & \multicolumn{3}{|c|}{$\mathrm{g}=0.6 \& \mathrm{a}=0.5$} & \multicolumn{3}{|c|}{$\mathrm{g}=0.9 \& \mathrm{a}=0.5$} \\
\hline & Empirical & Theoretical & PRE & Empirical & Theoretical & PRE & Empirical & Theoretical & PRE & Empirical & Theoretical & PRE \\
\hline$t_{m}$ & 0.0247 & 0.0234 & 100.00 & 0.0016 & 0.0024 & 100.00 & 0.0022 & 0.0085 & 100.00 & 0.0030 & 0.0189 & 100.00 \\
\hline$t_{S}$ & 0.0149 & 0.0138 & 169.18 & 0.0015 & 0.0020 & 119.61 & 0.0030 & 0.0052 & 163.22 & 0.0053 & 0.0112 & 169.23 \\
\hline$t_{G}$ & 0.0131 & 0.0125 & 186.59 & 0.0015 & 0.0019 & 127.08 & 0.0020 & 0.0048 & 178.99 & 0.0028 & 0.0101 & 186.59 \\
\hline$t_{K_{1}}$ & 0.0130 & 0.0125 & 187.04 & 0.0014 & 0.0019 & 129.79 & 0.0020 & 0.0047 & 182.83 & 0.0028 & 0.0100 & 189.20 \\
\hline$t_{K_{2}}$ & 2.0667 & 0.0579 & 40.41 & 0.0583 & 0.0325 & 7.51 & 0.1720 & 0.1271 & 6.70 & 0.4629 & 0.2854 & 6.63 \\
\hline$t_{1}$ & 0.0130 & 0.0125 & 186.59 & 0.0015 & 0.0019 & 127.08 & 0.0020 & 0.0048 & 178.99 & 0.0028 & 0.0101 & 186.59 \\
\hline$t_{2}$ & 0.0189 & 0.0176 & 133.22 & 0.0016 & 0.0024 & 102.52 & 0.0024 & 0.0066 & 129.68 & 0.0035 & 0.0142 & 133.24 \\
\hline \multicolumn{13}{|l|}{ Proposed } \\
\hline$t_{g}$ & 0.0102 & 0.0096 & 243.29* & 0.0015 & 0.0017 & 146.99* & 0.0020 & 0.0037 & 229.65 & 0.0028 & 0.0078 & 243.50* \\
\hline$t_{g_{1}}$ & 0.0102 & 0.0096 & 243.04 & 0.0067 & 0.0040 & 61.00 & 0.0102 & 0.0045 & 188.50 & 0.0139 & 0.0079 & 241.02 \\
\hline$t_{g 2}$ & 0.0116 & 0.0110 & 211.97 & 0.0023 & 0.0018 & 137.08 & 0.0044 & 0.0042 & 203.83 & 0.0073 & 0.0088 & 214.27 \\
\hline$t_{g 3}$ & 0.0109 & 0.0100 & 233.33 & 0.0022 & 0.0021 & 119.02 & 0.0041 & 0.0038 & 227.20 & 0.0068 & 0.0080 & 237.69 \\
\hline$t_{g 4}$ & 0.0129 & 0.0119 & 196.64 & 0.0052 & 0.0034 & 71.76 & 0.0065 & 0.0044 & 193.20 & 0.0073 & 0.0093 & 202.57 \\
\hline$t_{g 5}$ & 0.0112 & 0.0106 & 220.15 & 0.0018 & 0.0021 & 118.45 & 0.0029 & 0.0053 & 160.45 & 0.0041 & 0.0114 & 166.40 \\
\hline$t_{g 6}$ & 0.0137 & 0.0119 & 197.30 & 0.0037 & 0.0021 & 116.19 & 0.0099 & 0.0050 & 170.06 & 0.0192 & 0.0107 & 176.16 \\
\hline$t_{g 7}$ & 0.0119 & 0.0121 & 193.70 & 0.0016 & 0.0039 & 62.72 & 0.0022 & 0.0082 & 104.28 & 0.0029 & 0.0152 & 124.56 \\
\hline$t_{g 8}$ & 0.0116 & 0.0110 & 211.97 & 0.0023 & 0.0018 & 137.08 & 0.0044 & 0.0042 & 203.83 & 0.0073 & 0.0088 & 214.27 \\
\hline$t_{g_{9}}$ & 0.0109 & 0.0100 & 233.33 & 0.0022 & 0.0021 & 119.02 & 0.0041 & 0.0038 & 227.20 & 0.0068 & 0.0080 & 237.69 \\
\hline
\end{tabular}


Table 8. Simulation and empirical results at sample size $n=50$ for the MSEs and PREs of the estimators using population II $\left[N=1000, \rho_{x 1 y}=0.8706 \& \rho_{x 2 y}=0.8428\right]$

\begin{tabular}{|c|c|c|c|c|c|c|c|c|c|c|c|c|}
\hline \multirow{4}{*}{$\begin{array}{l}\mathrm{n}=50 \\
\text { Estimators }\end{array}$} & \multicolumn{12}{|c|}{ MSE Estimation using Population-II } \\
\hline & \multirow{2}{*}{\multicolumn{3}{|c|}{ Himmelfarb and Edgel Model }} & \multicolumn{9}{|c|}{ Proposed Model } \\
\hline & & & & \multicolumn{3}{|c|}{$\mathrm{g}=0.3 \& \mathrm{a}=0.5$} & \multicolumn{3}{|c|}{$\mathrm{g}=0.6 \& \mathrm{a}=0.5$} & \multicolumn{3}{|c|}{$\mathrm{g}=0.9 \& \mathrm{a}=0.5$} \\
\hline & Empirical & Theoretical & PRE & Empirical & Theoretical & PRE & Empirical & Theoretical & PRE & Empirical & Theoretical & PRE \\
\hline$t_{m}$ & 0.0734 & 0.1144 & 100.00 & 0.0097 & 0.0123 & 100.00 & 0.0119 & 0.0417 & 100.00 & 0.0135 & 0.0925 & 100.00 \\
\hline$t_{S}$ & 0.0328 & 0.0378 & 302.75 & 0.0083 & 0.0092 & 134.64 & 0.0166 & 0.0154 & 271.39 & 0.0284 & 0.0305 & 303.25 \\
\hline$t_{G}$ & 0.0292 & 0.0279 & 409.38 & 0.0080 & 0.0082 & 149.70 & 0.0102 & 0.0118 & 352.53 & 0.0122 & 0.0225 & 410.57 \\
\hline$t_{K_{1}}$ & 0.0292 & 0.0279 & 409.52 & 0.0079 & 0.0082 & 150.43 & 0.0102 & 0.0118 & 354.63 & 0.0122 & 0.0225 & 410.75 \\
\hline$t_{K_{2}}$ & 1.7912 & 0.4621 & 24.75 & 0.0858 & 2.7010 & 0.46 & 0.1479 & 10.8033 & 0.39 & 0.3636 & 24.3072 & 0.38 \\
\hline$t_{1}$ & 0.0292 & 0.0279 & 409.38 & 0.0080 & 0.0082 & 149.70 & 0.0102 & 0.0118 & 352.53 & 0.0122 & 0.0225 & 410.57 \\
\hline$t_{2}$ & 0.0445 & 0.0684 & 167.20 & 0.0095 & 0.0119 & 103.53 & 0.0130 & 0.0264 & 158.05 & 0.0168 & 0.0553 & 167.20 \\
\hline \multicolumn{13}{|l|}{ Proposed } \\
\hline$t_{g}$ & 0.0088 & 0.0063 & $1809.81 *$ & 0.0076 & 0.0063 & 195.56* & 0.0102 & 0.0041 & 1028.08* & 0.0122 & 0.0050 & $1845.51 *$ \\
\hline$t_{g_{1}}$ & 0.0089 & 0.0066 & 1725.19 & 0.0503 & 0.0256 & 48.07 & 0.0723 & 0.0110 & \begin{tabular}{|l|}
378.77 \\
\end{tabular} & 0.0841 & 0.0059 & 1559.19 \\
\hline$t_{g 2}$ & 0.0134 & 0.0181 & 633.33 & 0.0143 & 0.0629 & 19.58 & 0.0345 & 0.0372 & 112.17 & 0.0408 & 0.0210 & 439.45 \\
\hline$t_{g 3}$ & 0.0093 & 0.0069 & 1667.35 & 0.0189 & 0.0127 & 97.08 & 0.0304 & 0.0070 & 592.90 & 0.0414 & 0.0052 & 1774.66 \\
\hline$t_{g 4}$ & 0.0220 & 0.0228 & 501.45 & 0.0342 & 0.0205 & 60.07 & 0.0423 & 0.0093 & 446.90 & 0.0414 & 0.0162 & 570.04 \\
\hline$t_{g 5}$ & 0.0150 & 0.0135 & 846.01 & 0.0101 & 0.0584 & 21.10 & 0.0205 & 0.0380 & 109.78 & 0.0205 & 0.0370 & 249.96 \\
\hline$t_{g 6}$ & 0.0304 & 0.0269 & 425.20 & 0.0302 & 0.0101 & 121.62 & 0.0700 & 0.0156 & 268.42 & 0.1156 & 0.0310 & 297.97 \\
\hline$t_{g 7}$ & 0.0108 & 0.0134 & 856.78 & 0.0105 & 0.0292 & 42.15 & 0.0127 & 0.0468 & 89.15 & 0.0139 & 0.0674 & 137.26 \\
\hline$t_{g 8}$ & 0.0134 & 0.0181 & 633.33 & 0.0143 & 0.0629 & 19.58 & 0.0345 & 0.0372 & 112.17 & 0.0408 & 0.0210 & 439.45 \\
\hline$t_{g_{9}}$ & 0.0093 & 0.0069 & 1667.35 & 0.0189 & 0.0127 & 97.08 & 0.0304 & 0.0070 & 592.90 & 0.0414 & 0.0052 & 1774.66 \\
\hline
\end{tabular}


Table 9. Simulation and empirical results at sample size $n=100$ for the MSEs and PREs of the estimators using population II $\left[N=1000, \rho_{x 1 y}=0.8706 \& \rho_{x 2 y}=0.8428\right.$ ]

\begin{tabular}{|c|c|c|c|c|c|c|c|c|c|c|c|c|}
\hline \multirow[b]{4}{*}{ Estimators } & \multicolumn{12}{|c|}{ MSE Estimation using Population-II } \\
\hline & \multirow{2}{*}{\multicolumn{3}{|c|}{ Himmelfarb and Edgel Model }} & \multicolumn{9}{|c|}{ Proposed Model } \\
\hline & & & & \multicolumn{3}{|c|}{$\mathrm{g}=0.3 \& \mathrm{a}=0.5$} & \multicolumn{3}{|c|}{$\mathrm{g}=0.6 \& \mathrm{a}=0.5$} & \multicolumn{3}{|c|}{$\mathrm{g}=0.9 \& \mathrm{a}=0.5$} \\
\hline & Empirical & Theoretical & PRE & Empirical & Theoretical & PRE & Empirical & Theoretical & PRE & Empirical & Theoretical & PRE \\
\hline$t_{m}$ & 0.0734 & 0.1144 & 100.00 & 0.0097 & 0.0123 & 100.00 & 0.0119 & 0.0417 & 100.00 & 0.0135 & 0.0925 & 100.00 \\
\hline$t_{S}$ & 0.0328 & 0.0378 & 302.75 & 0.0083 & 0.0092 & 134.64 & 0.0166 & 0.0154 & 271.39 & 0.0284 & 0.0305 & 303.25 \\
\hline$t_{G}$ & 0.0292 & 0.0279 & 409.38 & 0.0080 & 0.0082 & 149.70 & 0.0102 & 0.0118 & 352.53 & 0.0122 & 0.0225 & 410.57 \\
\hline$t_{1}$ & 0.0292 & 0.0279 & 409.38 & 0.0080 & 0.0082 & 149.70 & 0.0102 & 0.0118 & 352.53 & 0.0122 & 0.0225 & 410.57 \\
\hline$t_{2}$ & 0.0445 & 0.0684 & 167.20 & 0.0095 & 0.0119 & 103.53 & 0.0130 & 0.0264 & 158.05 & 0.0168 & 0.0553 & 167.20 \\
\hline \multicolumn{13}{|l|}{ Proposed } \\
\hline$t_{g}$ & 0.0088 & 0.0063 & $1809.81 *$ & 0.0076 & 0.0063 & $195.56 *$ & 0.0102 & 0.0041 & 1028.08* & 0.0122 & 0.0050 & 1845.51* \\
\hline$t_{g 5}$ & 0.0150 & 0.0135 & 846.01 & 0.0101 & 0.0584 & 21.10 & 0.0205 & 0.0380 & 109.78 & 0.0205 & 0.0370 & 249.96 \\
\hline$t_{g 6}$ & 0.0304 & 0.0269 & 425.20 & 0.0302 & 0.0101 & 121.62 & 0.0700 & 0.0156 & 268.42 & 0.1156 & 0.0310 & 297.97 \\
\hline$t_{g 7}$ & 0.0108 & 0.0134 & 856.78 & 0.0105 & 0.0292 & 42.15 & 0.0127 & 0.0468 & 89.15 & 0.0139 & 0.0674 & 137.26 \\
\hline$t_{g 8}$ & 0.0134 & 0.0181 & 633.33 & 0.0143 & 0.0629 & 19.58 & 0.0345 & 0.0372 & 112.17 & 0.0408 & 0.0210 & 439.45 \\
\hline$t_{g 9}$ & 0.0093 & 0.0069 & 1667.35 & 0.0189 & 0.0127 & 97.08 & 0.0304 & 0.0070 & 592.90 & 0.0414 & 0.0052 & 1774.66 \\
\hline
\end{tabular}


Table 10. Simulation and empirical results at sample size $n=200$ for the MSEs and PREs of the estimators using population II $\left[N=1000, \rho_{x 1 y}=0.8706 \& \rho_{x 2 y}=0.8428\right]$

\begin{tabular}{|c|c|c|c|c|c|c|c|c|c|c|c|c|}
\hline \multirow[b]{4}{*}{ Estimators } & \multicolumn{12}{|c|}{ MSE Estimation using Population-II } \\
\hline & \multirow{2}{*}{\multicolumn{3}{|c|}{ Himmelfarb and Edgel Model }} & \multicolumn{9}{|c|}{ Proposed Model } \\
\hline & & & & \multicolumn{3}{|c|}{$\mathrm{g}=0.3 \& \mathrm{a}=0.5$} & \multicolumn{3}{|c|}{$\mathrm{g}=0.6 \& \mathrm{a}=0.5$} & \multicolumn{3}{|c|}{$\mathrm{g}=0.9 \& \mathrm{a}=0.5$} \\
\hline & Empirical & Theoretical & PRE & Empirical & Theoretical & PRE & Empirical & Theoretical & PRE & Empirical & Theoretical & PRE \\
\hline$t_{m}$ & 0.0305 & 0.0241 & 100.00 & 0.0016 & 0.0026 & 100.00 & 0.0023 & 0.0088 & 100.00 & 0.0035 & 0.0195 & 100.00 \\
\hline$t_{S}$ & 0.0104 & 0.0080 & 302.89 & 0.0014 & 0.0019 & 134.20 & 0.0033 & 0.0032 & 271.30 & 0.0076 & 0.0064 & 303.27 \\
\hline$t_{G}$ & 0.0075 & 0.0059 & 408.83 & 0.0013 & 0.0017 & 148.85 & 0.0022 & 0.0025 & 353.01 & 0.0034 & 0.0048 & 409.89 \\
\hline$t_{1}$ & 0.0075 & 0.0059 & 408.83 & 0.0013 & 0.0017 & 148.85 & 0.0020 & 0.0025 & 353.01 & 0.0031 & 0.0048 & 409.89 \\
\hline$t_{2}$ & 0.0174 & 0.0144 & 167.22 & 0.0016 & 0.0025 & 103.19 & 0.0025 & 0.0056 & 158.09 & 0.0044 & 0.0116 & 167.27 \\
\hline \multicolumn{13}{|l|}{ Proposed } \\
\hline$t_{g}$ & 0.0017 & 0.0013 & $1810.53 *$ & 0.0013 & 0.0013 & 194.74* & 0.0020 & 0.0009 & $1034.12 *$ & 0.0031 & 0.0011 & 1854.29* \\
\hline$t_{g 5}$ & 0.0041 & 0.0029 & 844.91 & 0.0016 & 0.0123 & 21.06 & 0.0032 & 0.0080 & 109.88 & 0.0055 & 0.0078 & 249.94 \\
\hline$t_{g 6}$ & 0.0092 & 0.0057 & 424.69 & 0.0045 & 0.0021 & 121.03 & 0.0130 & 0.0033 & 267.99 & 0.0349 & 0.0065 & 297.71 \\
\hline$t_{g 7}$ & 0.0018 & 0.0028 & 856.94 & 0.0017 & 0.0062 & 42.05 & 0.0025 & 0.0099 & 89.15 & 0.0037 & 0.0142 & 137.21 \\
\hline$t_{g 8}$ & 0.0059 & 0.0038 & 633.68 & 0.0021 & 0.0133 & 19.55 & 0.0054 & 0.0078 & 112.12 & 0.0115 & 0.0044 & 439.50 \\
\hline$t_{g 9}$ & 0.0017 & 0.0015 & 1660.69 & 0.0028 & 0.0027 & 97.00 & 0.0057 & 0.0015 & 593.92 & 0.0118 & 0.0011 & 1770.00 \\
\hline
\end{tabular}


Table 11. Simulation and empirical results at sample size $n=300$ for the MSEs and PREs of the estimators using population II $\left[N=1000, \rho_{x 1 y}=0.8706 \& \rho_{x 2 y}=0.8428\right]$

\begin{tabular}{|c|c|c|c|c|c|c|c|c|c|c|c|c|}
\hline \multirow{4}{*}{$\begin{array}{l}\mathrm{n}=\mathbf{3 0 0} \\
\text { Estimators }\end{array}$} & \multicolumn{12}{|c|}{ MSE Estimation using Population-II } \\
\hline & \multirow{2}{*}{\multicolumn{3}{|c|}{ Himmelfarb and Edgel Model }} & \multicolumn{9}{|c|}{ Proposed Model } \\
\hline & & & & \multicolumn{3}{|c|}{$\mathrm{g}=0.3 \& \mathrm{a}=0.5$} & \multicolumn{3}{|c|}{$\mathrm{g}=0.6 \& \mathrm{a}=0.5$} & \multicolumn{3}{|c|}{$\mathrm{g}=0.9 \& \mathrm{a}=0.5$} \\
\hline & Empirical & Theoretical & PRE & Empirical & Theoretical & PRE & Empirical & Theoretical & PRE & Empirical & Theoretical & PRE \\
\hline$t_{m}$ & 0.0161 & 0.0141 & 100.00 & 0.0011 & 0.0015 & 100.00 & 0.0015 & 0.0051 & 100.00 & 0.0022 & 0.0114 & 100.00 \\
\hline$t_{S}$ & 0.0052 & 0.0046 & 302.80 & 0.0010 & 0.0011 & 134.82 & 0.0022 & 0.0019 & 271.43 & 0.0047 & 0.0037 & 303.48 \\
\hline$t_{G}$ & 0.0037 & 0.0034 & 409.62 & 0.0009 & 0.0010 & 149.50 & 0.0014 & 0.0015 & 351.37 & 0.0020 & 0.0028 & 409.75 \\
\hline$t_{K_{1}}$ & 0.0036 & 0.0034 & 413.24 & 0.0009 & 0.0010 & 149.50 & 0.0013 & 0.0014 & 358.74 & 0.0019 & 0.0028 & 412.73 \\
\hline$t_{K_{2}}$ & 2.1126 & 0.0575 & 24.44 & 0.0760 & 2.2969 & 0.07 & 0.2156 & 9.1875 & 0.06 & 0.4797 & 20.6718 & 0.05 \\
\hline$t_{1}$ & 0.0037 & 0.0034 & 409.62 & 0.0009 & 0.0010 & 149.50 & 0.0014 & 0.0015 & 351.37 & 0.0020 & 0.0028 & 409.75 \\
\hline$t_{2}$ & 0.0096 & 0.0084 & 167.26 & 0.0011 & 0.0015 & 103.42 & 0.0017 & 0.0032 & 158.33 & 0.0027 & 0.0068 & 167.16 \\
\hline \multicolumn{13}{|l|}{ Proposed } \\
\hline$t_{g}$ & 0.0010 & 0.0008 & 1801.28* & 0.0009 & 0.0008 & 193.59* & 0.0014 & 0.0005 & $1026.00 *$ & 0.0020 & 0.0006 & $1830.65 *$ \\
\hline$t_{g_{1}}$ & 0.0010 & 0.0008 & 1734.57 & 0.0060 & 0.0066 & 22.78 & 0.0093 & 0.0014 & 380.00 & 0.0141 & 0.0007 & 1554.79 \\
\hline$t_{g 2}$ & 0.0028 & 0.0022 & 632.88 & 0.0017 & 0.0181 & 8.36 & 0.0037 & 0.0046 & 112.25 & 0.0064 & 0.0026 & 438.22 \\
\hline$t_{g 3}$ & 0.0010 & 0.0008 & 1672.62 & 0.0021 & 0.0069 & 22.01 & 0.0038 & 0.0009 & 589.66 & 0.0068 & 0.0006 & 1773.44 \\
\hline$t_{g 4}$ & 0.0032 & 0.0028 & 500.00 & 0.0045 & 0.0228 & 6.62 & 0.0055 & 0.0012 & 446.09 & 0.0069 & 0.0020 & 567.50 \\
\hline$t_{g 5}$ & 0.0020 & 0.0017 & 846.39 & 0.0012 & 0.0135 & 11.17 & 0.0022 & 0.0047 & 109.85 & 0.0032 & 0.0045 & 250.00 \\
\hline$t_{g 6}$ & 0.0048 & 0.0033 & 424.47 & 0.0033 & 0.0269 & 5.61 & 0.0094 & 0.0019 & 268.59 & 0.0200 & 0.0038 & 297.12 \\
\hline$t_{g 7}$ & 0.0011 & 0.0016 & 856.71 & 0.0012 & 0.0134 & 11.31 & 0.0016 & 0.0058 & 89.22 & 0.0023 & 0.0083 & 137.08 \\
\hline$t_{g 8}$ & 0.0028 & 0.0022 & 632.88 & 0.0017 & 0.0181 & 8.36 & 0.0037 & 0.0046 & 112.25 & 0.0064 & 0.0026 & 438.22 \\
\hline$t_{g 9}$ & 0.0010 & 0.0008 & 1672.62 & 0.0021 & 0.0069 & 22.01 & 0.0038 & 0.0009 & 589.66 & 0.0068 & 0.0006 & 1773.44 \\
\hline
\end{tabular}

